\title{
El fomento del emprendimiento desde la Universitat Politècnica de València: El caso-estudio de la Facultad de Administración y Dirección de Empresas.
}

Faustino Sarrión-Viñes ${ }^{a}$, Elies Seguí-Mas ${ }^{b}$ y Guillermina Tormo-Carbóc

${ }^{a}$ Universitat Politécnica de València fausarvi@ade.upv.es ${ }^{\mathrm{b}}$ Universitat Politécnica de València esegui@cegea.upv.es y ${ }^{\mathrm{c}}$ Universitat Politécnica de València gtormo@omp.upv.es.

\begin{abstract}
Currently the college graduates are facing a troubled labor market, with unemployment rate among those less than 25 years of $51.8 \%$ (EPA IV Trim 2014) becomes training and professional skills in a key factor for insertion into the Labour Market. One mechanism is the entrepreneurship, which manages to generate self-employment and strengthen the business.

Since universities have emerged multitude of initiatives in this regard, among the best known are the star-up or spin-off, in which the knowledge and use of new technology is clearly present. But besides the support of these initiatives the Universitat Politècnica de València (UPV) is committed to the inclusion in the curriculum of transversal skills in entrepreneurship, thus giving students the tools necessary to successfully undertake and overcome the scarcity of employment predominant in Spain.

Therefore, this study examines the mechanisms that are being in the UPV to provide students skills in entrepreneurship; in particular we analyze the case of the Faculty of Business Administration and Management (FADE-UPV).
\end{abstract}

Keywords: Entrepreneurship, UPV, generic competences, university, teaching.

\section{Resumen}

Actualmente los egresados universitarios se enfrentan a un convulso mercado laboral, con una tasa de paro, entre los menores de 25 años, del 51.8\% (EPA IV Trimestre 2014) lo que convierte la formación y las competencias profesionales en un factor clave para su inserción en el mercado laboral. Uno de los mecanismos que mayor impulso está tomando es el emprendimiento, el cuál consigue generar autoempleo además de 
El fomento del emprendimiento desde la Universitat Politècnica de València: El caso-estudio de la Facultad de Administración y Dirección de Empresas.

fortalecer el tejido empresarial.

Desde las universidades han surgido multitud de iniciativas al respecto, entre las más conocidas están las start-up o las spin-off, en la que el conocimiento y el uso de nuevas tecnología está claramente presente. Pero además del apoyo a estas iniciativas la Universitat Politècnica de València (UPV) está apostando por la inclusión en los planes de estudio de competencias transversales en emprendimiento, dotando así a los estudiantes de las herramientas necesarias para emprender con éxito y sortear la escasez de empleo predominante en España.

Por consiguiente, este estudio analiza los mecanismos que se están llevando a cado en la UPV para proporcionar a los estudiantes universitarios competencias en emprendimiento, más concretamente se describe el caso particular de la Facultad de Administración y Dirección de Empresas (FADE-UPV).

Palabras clave: Emprendimiento, UPV, competencias transversales, universidad, docencia.

\section{Introducción}

El convulso mercado laboral predominante en estos momentos en España, demanda profesionales altamente competentes que sean capaces de adaptarse rápidamente a los cambios. Por lo que disponer de competencias transversales en diversas materias se convierte en una tarea fundamental para conseguir la estabilidad laboral deseada. En los últimos años, el emprendimiento está tomando fuerza como dinamizador de la economía y generador de empleo, es por ello que se debe potenciar. Siendo necesario formar a los estudiantes universitarios de competencias específicas en emprendimiento para capacitarlos con las herramientas necesarias que les ayuden en su futura etapa profesional.

Las universidades de todo el mundo están apostando por el emprendimiento de sus jóvenes universitarios, incluyendo en sus planes de estudio y programas, el fomento del emprendimiento (Kuratko, 2005), este impulso no proviene solamente del ámbito universitario, sino que también en la formación pre-universitaria se están impulsando programas de educación empresarial (Peterman et al., 2003).

En el trabajo de Klofsten (2000) se apunta que la formación en emprendizaje debe ir acompañada de tres actividades básicas en las universidades:

- Creación y mantenimiento de una cultura emprendedora en la Universidad.

(cc) EY-NC-ND 2015, Universitat Politècnica de València

Congreso IN-RED (2015) 
- Cursos específicos en emprendimiento, a través de los cuales los estudiantes universitarios puedan adquirir los conocimientos que son necesarios para emprender.

- La realización de cursos específicos para aquellas personas que deseen crear su propia empresa.

En el informe GEM España (2014) analiza la formación en emprendimiento en España, el cual, describe la necesidad de impulsar esta formación para incrementar el Espíritu Emprendedor de la población española. En el informe GEM España (2014) describe las posibles apuestas, de la última reforma en educación, por promover la inclusión de la formación emprendedora en la totalidad de los distintos niveles educativos, a través de las siguientes vías (GEM España, 2014):

- Principalmente en los currículos de enseñanza no universitaria: El desarrollo transversal de la competencia "Sentido de la iniciativa y espíritu emprendedor".

- En educación secundaria, formación profesional y en bachillerato: Incluyendo la asignatura "Iniciativa Emprendedora".

- Finalmente en la enseñanza universitaria se ha optado por la anterior vía, con la inclusión de materias como "Creación de Empresas".

Aunque estas medidas aún están lejos de su objetivo deseado, apuntan la necesidad de actuar a corto plazo en (GEM España, 2014):

- Expansión de la cultura emprendedora más allá del ámbito educativo.

- La implicación de todo el cuerpo docente del sistema.

Es por ello, que las universidades, como agentes formadores han de anticiparse y dar respuesta a las transformaciones sociales y las exigencias que se plantean en el entorno (Concha et al., 2004). En este estudio se pretende analiza los mecanismos que se están llevando a cado en la UPV para proporcionar a los estudiantes universitarios competencias en emprendimiento, más concretamente se describe el caso particular de la Facultad de Administración y Dirección de Empresas (FADE-UPV).

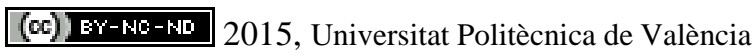

Congreso In-Red (2015) 
El fomento del emprendimiento desde la Universitat Politècnica de València: El caso-estudio de la Facultad de Administración y Dirección de Empresas.

\section{Objetivos}

El objetivo principal de la presente investigación es el análisis del papel de la UPV en el apoyo e impulso de la actividad emprendedora, a través del caso-estudio de la Facultad de Administración y Dirección de Empresas, más concretamente se persigue observar la existencia de:

- Docencia relacionada directamente con el emprendimiento.

- La realización de actividades vinculadas con el emprendimiento (seminarios, conferencias, ferias etc.).

- El papel de FADE el apoyo e impulso de la actividad emprendedora dentro de la UPV.

\section{Metodología}

La metodología que se ha utilizado en la presente investigación es un estudio del caso, más concretamente el estudio de FADE en el impulso de la actividad emprendedora de sus alumnos y egresados. El estudio del caso es una investigación empírica que analiza fenómenos en contextos de la vida real (Yin, 1994). Esta metodología suele utilizarse cuando los límites entre los fenómenos y sus contextos no son claramente evidentes (Yin, 1994).

La realización del estudio ha utilizado múltiples fuentes de evidencia (entrevista-encuesta y revisión de documentación). La encuesta se ha remitido a los participantes del ESPACIO EMPRENDE que utilizan o han utilizado las instalaciones en la facultad, con el objetivo de que aporten su visión sobre el papel que está llevando a cabo la Facultad de ADE. La información corporativa de la UPV así como la propia de la Facultad ha sido relevante para complementar los datos necesarios para el análisis.

\section{Recursos de la Facultad de Administración y Dirección de Empresas para el emprendimiento.}

En este apartado se van a analizar, en primer lugar la FADE, y seguidamente, se expondrán las distintas actividades (cursos, asignaturas, programas etc.) que se llevan a cabo para formar e impulsar las competencias de sus estudiantes y egresados en emprendimiento.

Entre las facultades de más reciente creación en la UPV (aprobación abril del 2000) se encuentra la Facultad de Administración y Dirección de Empresas (FADE- UPV). La cuál 
imparte los títulos conducentes a la obtención del Grado en Administración y Dirección de Empresas (ADE) y Grado en Gestión y Administración Pública (GAP). Actualmente los títulos de Licenciado en ADE y Diplomado en GAP se encuentran en extinción por la aplicación del Plan Boloña. También se imparten estudios de postgrados, Máster en Dirección Financiera y Fiscal (MDFF) y Máster en Gestión de Empresas, Productos y Servicios (MGEPS)-, además se puede acceder al programa de Doctorado en ADE, el cuál actualmente está gestionado por la Escuela de Doctorado de la UPV (UPV, 2015 a).

\subsection{Espacio Emprende FADE.}

La Universitat Politècnica de València, a través del Instituto IDEAS UPV, ha lanzado el programa emprendedor "StartUPV", el cual pretende ser un nexo de unión entre la universidad y los emprendedores. Para ello, la UPV pone a disposición de los emprendedores, start-ups, alumnos, egresados y mentores una red de espacios de trabajo y co-creación para así desarrollar los proyectos e iniciativas empresariales (UPV, 2015 b).

Los participantes de este programa pueden utilizar instalaciones de la universidad, las cuales están situadas en la Ciudad Politécnica de la Innovación (CPI), la casa del alumno y en cada una de las escuelas, facultades y campus de la UPV (Espacios [EMPRENDE]). La UPV restringe los espacios de trabajo a los alumnos y titulados (alumni) (UPV, 2015 b).

En la FADE, en la planta 4, se dispone de un Espacio EMPRENDE. En este espacio se ceden y comparten materiales, mobiliario, recursos y tiempo, de forma gratuita. Se han identificado en FADE cinco proyectos emprendedores, siendo estos (1) Planarte, (2) Vitabelia Market, (3) Neuron SW y (4) MIOTtech, (5) Vitcord.

La empresa Planarte está impulsada por un antiguo alumno de Administración y Dirección de Empresas. Esta empresa es una web de viajes, en la que sus clientes a través de juegos sociales pueden ganar puntos que son canjeables por descuentos en viajes.

Vitabelia Market es una Sociedad de Responsabilidad Limitada Unipersonal. Esta empresa es marketplace de productos y servicios infantiles, la cual está orientada a los padres.

Por lo que respecta a Neuron SW, es un empresario individual, proveedor de hosting avanzado, el cuál es muy fácil de usar y para todo el mundo. Capa por encima de los hosting actuales del mercado, ofreciendo un hosting.

La empresa MIOTech se crea con el propósito de unir el mundo digital con el mundo real. Esta start-up, que recientemente se ha constituido como sociedad limitada, se dedica al desarrollo de productos de hardware conectados a internet (IOT) (MIOTtech, 2015). Según sus fundadores próximamente su producto, un contador social que registra el impacto que 
El fomento del emprendimiento desde la Universitat Politècnica de València: El caso-estudio de la Facultad de Administración y Dirección de Empresas.

tiene un negocio en las redes sociales y lo muestra directamente en el contador, se lanzará a nivel mundial, en el que esperan ofrecer su producto a un mercado mucho más amplio.

Finalmente, la empresa Vitcord, la cual actualmente se ha trasladado a la CPI de la UPV, ofrece a sus clientes una aplicación de creación y grabación de un video, a partir de clips de amigos. Esta start-up ha ganado varios premios, como "Startup Junios 5U-CV Talent 2014" o "Primer accésit Valencia Emprende 2014" (Vitcord, 2015).

\subsection{Cátedra Bancaja Jóvenes-Emprendedores}

La Cátedra Bancaja Jóvenes Emprendedores, la cual lleva a cabo sus actividades a través de FADE, el Centro de Especialización en Gestión de Empresas Agroalimentarias (CEGEA) y el Instituto para la Creación y Desarrollo de Empresas Programa Ideas, tiene como objetivo el fomento del espíritu y la vocación empresarial (UPV, 2015 c).

La Cátedra organiza y celebra multitud de actividades formativas de diversa índole, entre las que destaca la organización de jornadas, conferencias, clases magistrales, seminarios, clases o ciclos de conferencias. Además apoya la publicación de monografías sobre el objeto de la Cátedra, desarrolla actividades culturales y de difusión docente e investigadora, colabora en proyectos en los que Bancaja tenga convenios con universidades, desarrolla programas de investigación que están vinculados con la actividad emprendedora, así como, impulsa, en la zona de actuación e influencia de Bancaja, la creación de empresas y el desarrollo del emprendedor (UPV, 2015 c).

\subsection{Cátedra Cultura Directiva y Empresarial}

La Cátedra Cultura Directiva y Empresarial está adscrita a la Facultad de Administración y Dirección de Empresas, la cual está promovida por el Consejo Social e ideas, con el objetivo de fomentar la cultura directiva y empresarial en los alumnos de la Universidad. Así como aumentar el conocimiento, las relaciones entre la empresa y la Universidad, impulsar y crear actividades innovadoras y complementarias que estén relacionadas con la función directiva y el mundo de la empresa (UPV, 2015 d).

Entre las diversas actividades que se organizan en la Cátedra resalta la jornada campus [EMPRENDE], el cual es un curso dirigido principalmente a personas con un espíritu emprendedor. Con un elevado carácter práctico, combina clases teóricas y talleres, así se van generando y desarrollando los distintos proyectos que hayan sido previamente seleccionados. El curso introducción al mundo de la empresa, el cuál es su V edición consiguió 40 alumnos matriculados, 20 ponentes, 11 entidades patrocinadoras, 8 sesiones 
formativas y 2 jornadas técnicas (IDEAS, 2015). Esto son algunos de los ejemplos de las actividades que se llevan a cabo en la Cátedra para impulsar el emprendimiento.

\subsection{Subdirección de emprendimiento}

La subdirección depende de la Dirección Delegada de Emprendimiento y Empleo de la UPV. El Vicerrector/Director Delegado de Emprendimiento y Empleo es el responsable de las actividades e iniciativas de la UPV que están dirigidas a la inserción laboral de los graduados, seguimiento de la acción de Servipoli, y la realización de prácticas en empresas. Además se encargan de fomentar y gestionar las iniciativas emprendedoras que se crean en la Universitat dirigidas a la creación de empresas innovadoras (UPV, 2015 e).

La misión de la subdirección es facilitar así como promocionar la formación en emprendimiento y el espacio emprende, que forma parte del ecosistema emprendedor de la Universitat (UPV, 2015).

\subsection{Formación en FADE}

En la FADE actualmente dispone de diversas titulaciones, aunque algunas de ellas debido al nuevo plan académico están en extinción, (1) Licenciatura ADE (en extinción) (2) Diplomatura en GAP (en extinción), (3) Grado en ADE, (4) Doble grado en Administración y Dirección de Empresas e Ingeniería de Tecnologías y Servicios de Telecomunicación, (5) Doble grado en Administración y Dirección de Empresas e Ingeniería Informática (inicio septiembre 2016), (6) Máster en Dirección Financiera y Fiscal, (7) Máster Oficial en Gestión de Empresas, Productos y Servicios. La búsqueda de asignaturas que en su denominación incluyan el término emprendedores, emprendedor o emprendimiento ha evidenciado que solamente en el grado de ADE se está ofreciendo una asignatura Emprendedores y Creación de Empresas, la cuál es una optativa a las prácticas en empresas. El escaso grado de asignaturas que contemplan el emprendimiento como enfoque principal de la enseñanza evidencia la necesidad de incluir asignaturas sobre emprendimiento en el resto de titulaciones.

Por lo que respecta a la formación de los alumnos en cursos o seminarios sobre emprendimiento, diverge a la tónica seguida en las asignaturas, ya que anualmente se realizan multitud de cursos y seminarios, que si bien organizados por las cátedras o desde la propia facultad fomentan el emprendimiento entre los alumnos.

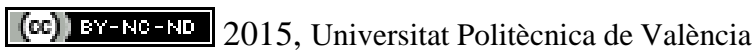

Congreso In-Red (2015) 
El fomento del emprendimiento desde la Universitat Politècnica de València: El caso-estudio de la Facultad de Administración y Dirección de Empresas.

\subsection{El caso-estudio.}

Anteriormente se han revisado los distintos recursos que la Facultad destina a la formación e impulso de la actividad emprendedora entre sus estudiantes. Con el objetivo de disponer de una visón práctica, se realizó una encuesta-entrevista semiestructurada a los fundadores de la empresa MIOTtech, dado que actualmente está utilizando las instalaciones del ESPACIO EMPRENDE y así, aporten su visión sobre el papel que se está llevando a cabo la Facultad de ADE y la UPV. Además se remitió la encuesta vía correo a las restantes empresas que habían utilizado estas instalaciones, con lo que se obtuvo la respuesta de Vitabelia. Finalmente, dos respuestas válidas se obtuvieron del caso de MIOTtech, y una de Vitabelia.

La empresa MIOTech se crea por un equipo de dos jóvenes emprendedores estudiantes de la Universitat Politècnica de València, con el propósito de unir el mundo digital con el mundo real. Esta empresa de reciente creación se dedica al desarrollo de productos de hardware conectados a internet (IOT). Ofrecen un contador social, el cuál registra el impacto que tiene aquello que se quiere medir en las redes sociales, mostrándolo directamente (MIOTtech, 2015). Vitabelia Market es una Sociedad de Responsabilidad Limitada Unipersonal. Esta empresa es marketplace de productos y servicios infantiles, la cual está orientada a los padres.

La realización de una encuesta-entrevista semiestructurada ha MIOTech y la encuesta a Vitabelia ha permitido observar las opiniones de los fundadores de las start-up sobre los recursos y materiales que la FADE y la UPV ponen a su disposición.

El estudio del perfil de los emprendedores, evidencia que se trata que jóvenes emprendedores, con un rango de edad de los 25 a los 31 años, de nacionalidad española y con estudios superiores. Ambas empresas recientemente se han constituido como sociedad limitada (2015 y 2014), y en el momento de su creación dos de los emprendedores eran estudiantes, en cambio en el tercer caso estaba en proceso de cambio de empresa dentro del mismo sector en el que estaba trabajando.

Solo uno de los fundadores ha recibido formación específica para el emprendimiento, pero esta no había sido recibida por cursos o seminarios impartidos por la FADE, sino por la UPV. Según los emprendedores el papel de la UPV y la FADE en el proceso de creación de su empresa ha sido escaso, resaltando que la principal aportación en la creación de la empresas desde la UPV era la cesión de instalaciones, el contacto con empresas, la ayuda administrativa para la realización de trámites y la información de fuentes de financiación. En cuanto a FADE la cesión de instalaciones es el principal recurso proporcionado en la creación de la empresa. 
La disponibilidad del Espacio EMPRENDE fue el recurso más importante para los emprendedores en FADE, seguido de la subdirección de emprendimiento y la cátedra de Cultura Empresarial. Por lo que respecta a la UPV, el espacio Emprendedor STARTUPV y el Instituto IDEAS son los recursos cuya disponibilidad se considera más importantes, seguidos de cursos y jornadas realizados en la UPV.

Ninguno de los encuestados ha cursado la asignatura "Emprendedores y Creación de Empresas". Dos de los encuestados coinciden en señalar que la impartición de asignaturas sobre emprendimiento se debería realizar como una asignatura completa, y no incluir el emprendimiento en varias asignaturas a lo largo del grado. En cambio el tercer encuestado discrepa en este aspecto, el cual considera que una de las principales debilidades es el enfoque actual del emprendimiento. Apuntando que las asignaturas sobre emprendimiento no deben plantearse como una asignatura tradicional. Según el encuestado el emprendimiento no es una lección, sino un valor, una competencia, una manera de ser y de entender la vida profesional.

En cuanto a debilidades y fortalezas que existen en FADE para el emprendimiento, se apuntó la adecuada disposición del espacio, puesto que es un lugar de trabajo tranquilo y disponen de asesoría sobre emprendimiento al abasto, apuntando que algunos recursos como el acceso a internet podría mejorar.

\section{Conclusiones.}

La situación económica y social actual, en la que las tasas de paro están en niveles insostenibles a largo plazo, el emprendimiento se postula como una posible salida a esta situación. Analizar el papel de la Facultad de Administración y Dirección de Empresas en este proceso ha sido el objeto de este estudio.

El análisis de los diversos recursos que la UPV y la FADE disponen ha evidenciado el activo papel que está desarrollando la Universitat por apostar por el emprendimiento de sus estudiantes y egresados.

El estudio de la FADE y las encuestas realizadas, ha evidenciado que la inclusión en los planes de estudio del emprendimiento todavía no se está aplicando a todas las titulaciones, puesto que solo el grado de ADE, y cómo opción optativa a las prácticas, ofrece una asignatura íntegra sobre emprendimiento. Según dos de los tres encuestados preferirían disponer de asignaturas completas sobre emprendimiento, y no como partes de alguna asignatura, por lo que se debería incluir alguna asignatura sobre emprendimiento en los restantes planes de estudio. Aunque el tercer encuestado realiza una aportación muy interesante para la reflexión en cuanto que no se debe realizar asignaturas tradicionales,

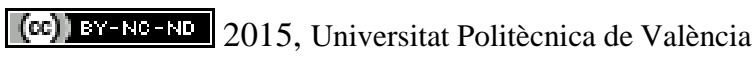

Congreso In-Red (2015) 
El fomento del emprendimiento desde la Universitat Politècnica de València: El caso-estudio de la Facultad de Administración y Dirección de Empresas.

sino más bien, dar ganas y generar curiosidad en los alumnos y posteriormente proveer a los alumnos de herramientas y espacios donde puedan desarrollar sus inquietudes.

Como se ha podido observar anteriormente, tanto en la UPV como en FADE la realización de cursos, seminarios y jornadas sobre emprendimiento es constante y proviene de diversos organizadores (cátedras, cfp, facultad etc). Por lo que la apuesta desde la formación vía cursos o jornadas parece adecuada. Los entrevistados apuntaron a la disponibilidad de cursos y jornadas realizados en la UPV es importante.

En conclusión, pese a no existir un amplio abanico de asignatura en FADE que estudien el emprendimiento como objeto principal, la realización de cursos, seminarios, espacios y estructuras que dispone, evidencia el activo papel que la FADE y la UPV están desarrollando para impulsar el emprendimiento entre sus estudiantes y egresados.

\section{Referencias.}

CONCHA, A., ALVAREZ, C., y SÁEZ, E. (2004). "Programa de formación de emprendedores en la universidad de concepción” en Roig, S.; Ribeiro, D.; Torcal, R.; De La Torre, A, , 311-322.

GLOBAL ENTREPRENEURSHIP MONITOR (2014). Informe GEM España 2014. Global entrepreneuship Association. <http://www.gem-spain.com/wpcontent/uploads/2015/04/GEM_es_2014.pdf> [Consulta: 10 de mayo de 2015].

IDEAS (2015). Curso de iniciación al mundo de la empresa.. <http://issuu.com/institutoideas/docs/dossier> [Consulta: 26 de mayo de 2015]

KLOFSTEN, M. (2000). "Training entrepreneurship at universities: A swedish case" en Journal of European Industrial Training, 24(6), 337-344.

KURATKO, D. F. (2005). “The emergence of entrepreneurship education: Development, trends, and challenges" en Entrepreneurship Theory and Practice, 29(5), 577-598.

MIOTtech (2015). El poder de MIOT . < http://miottech.com/ > [Consulta: 27 de mayo de 2015] 
PETERMAN, N. E., y KENNEDY, J. (2003). "Enterprise education: Influencing students' perceptions of entrepreneurship" en Entrepreneurship Theory and Practice, 28(2), 129-144.

UNIVERSITAT POLITÈCNICA DE VALÈNCIA (2015 a). Facultad de Administración y Dirección de Empresas. <http://www.emeraldinsight.com/doi/full/10.1108/03090590010373325 > [Consulta: 15 de mayo de 2015]

UNIVERSITAT POLITÈCNICA DE VALÈNCIA (2015 b). Programa STARTUPV. <http://www.ideas.upv.es/servicios/startupv/ > [Consulta: 15 de mayo de 2015]

UNIVERSITAT POLITÈCNICA DE VALÈNCIA $(2015$ c). Cátedra BANCAJA Jóvenes Emprendedores UPV . <http://www.upv.es/contenidos/CBANCAJA/indexc.html > [Consulta: 16 de mayo de 2015]

UNIVERSITAT POLITÈCNICA DE VALÈNCIA (2015 d). Cátedra Cultura Directiva y Empresarial. <http://www.upv.es/contenidos/CCDE/infoweb/ccde/info/907469normalc.html> [Consulta: 20 de mayo de 2015]

UNIVERSITAT POLITÈCNICA DE VALÈNCIA (2015 e). Dirección Delegada de Emprendimiento y Empleo. < http://www.upv.es/entidades/DE2/indexc.html> [Consulta: 27 de mayo de 2015]

VITCORD (2015). Vitcord. < http://vitcord.com/ > [Consulta: 27 de mayo de 2015]

YIN, R. K. (2013). Case study research: Design and methods. Sage publications. 